\title{
Comparison of Outcomes for Laser Trabeculoplasty after Kahook Dual Blade Goniotomy Versus in Goniotomy-Naive Eyes
}

\author{
Junsang Cho (D) - Daniel Hogan · Muhammad Salim • \\ Eli L. Pratte · Joshua King · Riley Bylund · Matthew T. Hirabayashi • \\ Jella A. An
}

Received: June 17, 2021 / Accepted: July 12, 2021 / Published online: July 24, 2021

(c) The Author(s) 2021

\section{ABSTRACT}

Introduction: This was a comparison of laser trabeculoplasty (LTP) outcomes in eyes with prior Kahook Dual Blade (KDB) goniotomy versus in goniotomy-naive control eyes.

Methods: This was a retrospective matched comparative case series. We identified a cohort of patients undergoing LTP between February 2017 and July 2020 at University of Missouri. Patients were grouped by history of KDB goniotomy versus goniotomy-naivety as a control group. Inclusion criteria included age at least 18 years, minimum of 6 months follow-up after LTP, and minimum period of 6 months between KDB goniotomy and LTP. All KDB procedures were combined with uncomplicated phacoemulsification. Patients who had any additional intraocular pressure (IOP)-lowering procedures between KDB goniotomy and LTP

Supplementary Information The online version contains supplementary material available at https:// doi.org/10.1007/s40123-021-00378-7.

J. Cho - D. Hogan · M. Salim · E. L. Pratte · J. King ·

R. Bylund · M. T. Hirabayashi · J. A. An ( $₫)$

University of Missouri School of Medicine,

Columbia MO USA

e-mail: anja@health.missouri.edu

R. Bylund · M. T. Hirabayashi · J. A. An

Department of Ophthalmology, Mason Eye

Institute, University of Missouri, 3215 Wingate

Court, Columbia, MO 65201, USA were excluded. Patients in the control group received a single LTP procedure.Primary outcome consisted of the comparison of LTP success, defined as IOP reduction of at least $20 \%$ or reduction of glaucoma medications from preLTP baseline. Secondary outcomes included IOP and medication reduction from pre-LTP baseline.

Results: Twenty-one eyes of 19 patients with history of KDB goniotomy and 42 eyes of 36 control patients without previous angle or laser procedures were included. Baseline characteristics including age, gender, ethnicity, type and severity of glaucoma, baseline IOP, and baseline medications were matched between groups. The LTP success rate was higher in the control group, but was not statistically significant (64\% vs $57 \%, p=0.58$ ). IOP reduction was only significant in the control eyes $(2.50 \pm 4.0 \mathrm{mmHg}$, $p=0.01$ vs $2.35 \pm 4.7 \mathrm{mmHg}, p=0.08$ ). The number of glaucoma medications was not significantly reduced in either group.

Conclusion: LTP may have a limited IOP- and medication-lowering effect in eyes with a history of KDB goniotomy compared to goniotomy-naive eyes.

Keywords: Glaucoma; Kahook Dual Blade; Laser trabeculoplasty; MIGS 


\section{Key Summary Points}

Why carry out this study?

As minimally invasive glaucoma surgery (MIGS) gains popularity as treatment for glaucoma, there have been several studies that outline postoperative outcomes. Non-incisional therapies such as selective laser trabeculoplasty (SLT) have been used as adjunct therapy for glaucoma in patients with history of MIGS. This study is the first to compare outcomes for patients who have received SLT after Kahook Dual Blade (KDB) goniotomy to a control group who only received SLT.

\section{What was learned from this study?}

Our results show that eyes with prior KDB goniotomy had limited IOP- or medication-lowering response to subsequent LTP performed. However, further studies must be performed to confirm our findings. can be limited and unpredictable [9]. Hirabayashi et al. reported that $9.5 \%$ of eyes that underwent $\mathrm{KDB}$ goniotomy for all levels of glaucoma severity needed additional IOP-reducing procedures including trabeculoplasty (6.8\%), micropulse cytophotocoagulation $(1.4 \%)$, and filtering surgery (1.4\%) [3].

Laser trabeculoplasty (LTP) is frequently offered in patients who had inadequate response to an angle surgery in attempt to further lower IOP or reduce medication burden. King et al. recently reported that the outcome of LTP is not predictive of the outcome of subsequent $\mathrm{KDB}$ goniotomy, and $\mathrm{KDB}$ goniotomy surgery remains to be effective even in eyes with failed LTP [10]. However, the question of whether LTP could successfully lower IOP in eyes with history of KDB goniotomy still exists, and to our knowledge there is no published data that addresses this question. Our primary goal was to compare the IOP- and medication-lowering outcome of LTP after KDB goniotomy compared to that in goniotomy-naive control eyes.

\section{METHODS}

\section{Patient Selection and Data Collection}

This was a retrospective analysis of data from patients who underwent LTP with history of KDB goniotomy and phacoemulsification compared to goniotomy-naive eyes at the University of Missouri in Columbia from February 1, 2017 to July 31,2020 . All study related analyses were approved by the institutional review board of the University of Missouri Columbia School of Medicine (Columbia, MO, USA, IRB \#2019849). This study adhered to all tenets of the Declaration of Helsinki and its later amendments or comparable ethical standards. A waiver of consent and Health Insurance Portability and Accountability Act (HIPAA) authorization was granted in accordance with the regulations of the Common Rule and HIPAA Privacy Rule.

Data was drawn from the charts of adult patients with early to advanced primary or secondary open-angle glaucoma. Glaucoma severity was defined using the International 
Table 1 Demographic characteristics of both prior KDB goniotomy and goniotomy-naive eyes

\begin{tabular}{|c|c|c|c|}
\hline Subject-level parameters & Prior KDB goniotomy $(n=19)$ & Goniotomy-naive $(n=36)$ & $p$ value \\
\hline Age (years), mean $\pm S D$ & $69.40 \pm 8.1$ & $68.20 \pm 7.9$ & 0.61 \\
\hline \multicolumn{4}{|l|}{ Gender, $\%(n)$} \\
\hline Female & $68.42(13)$ & $61.11(22)$ & 0.74 \\
\hline Male & $31.58(6)$ & $38.89(14)$ & \\
\hline \multicolumn{4}{|l|}{ Ethnicity, \% $(n)$} \\
\hline Caucasian & $89.95(17)$ & $88.89(32)$ & 0.94 \\
\hline Other & $10.52(2)$ & $11.11(4)$ & \\
\hline Eye-level parameters & Prior KDB goniotomy & LTP only $(n=42)$ & $p$ value \\
\hline \multicolumn{4}{|l|}{ Glaucoma diagnosis, $\%(n)$} \\
\hline Primary open-angle & $76.19(16)$ & $83.33(35)$ & 0.13 \\
\hline Normal tension & $9.52(2)$ & $11.90(5)$ & \\
\hline Combined mechanism & $9.52(2)$ & $0.0(0)$ & \\
\hline Pseudoexfoliatative & $4.76(1)$ & $0.0(0)$ & \\
\hline Traumatic & $0.0(0)$ & $4.76(2)$ & \\
\hline \multicolumn{4}{|l|}{ Glaucoma severity, \% (n) } \\
\hline Mild & $33.33(7)$ & $26.19(11)$ & 0.87 \\
\hline Moderate & $9.52(2)$ & $14.29(6)$ & \\
\hline Severe & $57.14(12)$ & $59.52(25)$ & \\
\hline Pre-laser IOP $(\mathrm{mmHg})$, mean \pm SI & $19.52 \pm 4.7$ & $17.40 \pm 4.0$ & 0.09 \\
\hline Pre-laser medications, mean $\pm S D$ & $1.62 \pm 1.3$ & $1.74 \pm 1.3$ & 0.74 \\
\hline \multicolumn{4}{|l|}{ Type of LTP, \% (n) } \\
\hline MLT & $85.71(18)$ & $73.81(31)$ & 0.35 \\
\hline SLT & $14.2(3)$ & $26.19(11)$ & \\
\hline
\end{tabular}

IOP intraocular pressure, $L T P$ laser trabeculoplasty, $K D B$ Kahook Dual Blade

*Significance determined using chi-square, Fisher's exact, Student's $t$ test, and Mann-Whitney $U$ test

Classification of Disease (ICD)-10 classification [11]. The indications for both LTP and KDB goniotomy were the need for IOP or medication reduction due to confirmed progression at baseline IOP or difficulty with medication adherence. All patients who received KDB goniotomy had a visually significant cataract that required removal as a combined procedure. Only subjects who had a minimum of 6 months of follow-up after LTP and 6 months interval between KDB goniotomy and LTP were included. All patients who required IOP-lowering procedures between KDB goniotomy and LTP were excluded. Both selective laser trabeculoplasty (SLT) and micropulse laser trabeculoplasty (MLT) were considered equivalent and included as part of our analysis for LTP procedures, whereas those with a history of argon 
laser trabeculoplasty (ALT) were excluded $[12,13]$.

A total of 21 eyes of 19 patients were included in the prior KDB goniotomy combined with phaco group and 42 goniotomy-naive eyes from 36 control patients were included in the control group. Eyes in the control group had no additional prior or subsequent LTP performed. The control group did not include pseudophakic eyes as there have been studies published that show SLT has comparable effect in pseudophakic versus phakic eyes [14]. Each eye was considered independently. Baseline characteristics including age, gender, ethnicity, glaucoma type and severity, IOP, and the number of glaucoma medications were matched between groups (Table 1). For outcome measures, we recorded IOP and number of medications at each postoperative visit. Skilled technicians and treating ophthalmologists were blinded to the study during assessment of IOP and medication use after surgery.

\section{Surgical Procedures}

Among LTP procedures, all MLT was performed with an IQ 532TM laser (Iridex Corporation, Mountain View, CA, USA) with $532 \mathrm{~nm}$ wavelength, $15 \%$ duty cycle $(500 \mathrm{~Hz})$, duration of $300 \mathrm{~ms}$, spot size $300 \mu \mathrm{m}$, and $1000 \mathrm{~mW}$ of power over $360^{\circ}$ of angle per procedure. SLT was performed with $532 \mathrm{~nm}$ a frequency-doubled Q-switched Nd:YAG laser with fixed 3 ns pulse and $400 \mu \mathrm{m}$ spot size, and variable energy between 0.6 and $1.4 \mathrm{~mJ}$ per pulse, titrated to visible microbubble, over $360^{\circ}$ of angle per procedure. No additional ocular hypotensive medication was given at the time of laser, unless IOP spike was noted at $1 \mathrm{~h}$ post laser. IOP was checked with a Goldmann applanation tonometer by a skilled technician or the treating ophthalmologist. Visual acuity and slit lamp examination were performed at 2 weeks, 2 months, and 6 months follow-up. All patients were kept under the same medications until seen 2 months post-LTP, when they were adjusted on the basis of the target IOP and sign of progression determined by the treating ophthalmologist.

KDB goniotomy was performed according to the KDB's Instructions for Use [15]. Procedures began with a temporal near-clear $2.4 \mathrm{~mm}(\mathrm{~mm})$ incision into the anterior chamber. Cohesive viscoelastic was injected into the anterior chamber. The patient's head was positioned, and microscope adjusted to allow visualization of the nasal angle using a Swam-Jacob-style gonio prism. A strip of TM spanning approximately 4 clock hours was then excised circumferentially and was removed with microforceps. Phacoemulsification was performed after KDB goniotomy in typical fashion with intraocular

Table 2 IOP and medication reduction after 6 months

\begin{tabular}{|c|c|c|c|}
\hline & Prior KDB goniotomy & Goniotomy-naive & $p$ value \\
\hline Pre-laser IOP $(\mathrm{mmHg})$, mean $\pm S D$ & $19.52 \pm 4.7$ & $17.40 \pm 4.0$ & 0.09 \\
\hline Post-laser IOP at 6 months $(\mathrm{mmHg})$, mean \pm SD ( $p$ value $)$ & $17.35 \pm 4.8(0.08)$ & $15.93 \pm 3.37\left(0.01^{*}\right)$ & 0.53 \\
\hline IOP reduction at 6 months $(\mathrm{mmHg})$, mean $\pm \mathrm{SD}$ & $2.35 \pm 4.7$ & $2.50 \pm 4.0$ & 0.81 \\
\hline IOP reduction $\%$ at 6 months, mean $\pm S D$ & $6.41 \pm 22.9$ & $13.62 \pm 18.3$ & 0.22 \\
\hline Pre-laser medications, mean $\pm S D$ & $1.62 \pm 1.3$ & $1.74 \pm 1.3$ & 0.74 \\
\hline Post-laser medications 6 months, mean \pm SD ( $p$ value) & $1.47 \pm 1.2(0.47)$ & $1.58 \pm 1.3(0.27)$ & 0.78 \\
\hline Post-laser medication reduction at 6 months, mean $\pm S D$ & $0.29 \pm 1.6$ & $0.11 \pm 0.9$ & 0.62 \\
\hline Post-laser medication reduction $\%$ at 6 months, mean $\pm S D$ & $17.31 \pm 64.5$ & $18.59 \pm 38.9$ & 0.94 \\
\hline
\end{tabular}

IOP intraocular pressure, $L T P$ laser trabeculoplasty, KDB Kahook Dual Blade

*Significance determined using Mann-Whitney $U$ test, Welch's $T$ test, and Wilcoxon rank sign test with alpha value less than 0.05 
Table 3 Success rates of prior KDB goniotomy versus goniotomy-naive eyes after 6 months

\begin{tabular}{llll}
\hline & Success & Failure & $\boldsymbol{p}$ value \\
\hline Prior KDB goniotomy, \% $(n)$ & $57.14(12)$ & $42.86(9)$ & 0.58 \\
Goniotomy-naive, \% $(n)$ & $64.29(27)$ & $35.71(15)$ & \\
\hline
\end{tabular}

LTP laser trabeculoplasty, $K D B$ Kahook Dual Blade

${ }^{*}$ Significance determined using chi-square test and $\alpha$ value $<0.05$

lens implantation. Viscoelastic was completely removed from the anterior chamber, and eyes were filled with balanced salt solution and intracameral moxifloxacin to achieve IOP in the mid-20s to minimize blood reflux at the conclusion of surgery. Preoperative IOP-lowering medications were continued in the postoperative period, and reduced only if target IOP was achieved and steroid was discontinued by no more than one medication per visit.

\section{Primary Outcome}

Success of LTP was defined as 6 month IOP reduction of at least $20 \%$ or medication reduction from pre-LTP baseline with no additional IOP-lowering interventions, including repeat LTP, within 6 months following the procedure. For secondary outcomes, we compared IOP and medication reduction at 6 months post-LTP compared to pre-LTP in both cohorts.

\section{Statistical Analysis}

A sample size calculation assumed of a mean postoperative IOP $11.83 \pm 2.21 \mathrm{mmHg}$ based on the available data in the literature [16]. At a power of $80 \%$ and an alpha level of $5 \%$, we estimated that a group size of 19 patients in each treatment arm would allow for the detection of a difference of $2 \mathrm{mmHg}$ or higher. Using success rates to calculate sample size was deferred because of the novelty of our study.

Baseline characteristics and IOP and medication reduction between the two cohorts were compared using chi-square, Fisher's exact, Student's $t$ test, and Mann-Whitney $U$ test. Success rates were compared using chi-square test. IOP differences between groups were compared using Mann-Whitney $U$ and Welch's $T$ test. IOP differences within groups across time were compared with Wilcoxon rank sign test. A $p$ value less than 0.05 was considered statistically significant.

\section{RESULTS}

Baseline patient characteristics were comparable between the prior KDB goniotomy and goniotomy-naive cohorts (Table 1). Subjects were evenly divided among men and women, mostly Caucasian, mean age of 70 , and diagnosed with severe primary open-angle glaucoma (POAG). Average duration between KDB goniotomy and LTP was $328.40 \pm 193.8$ days.

At 6 months, LTP was successful in $57.14 \%$ (12/21 eyes) of prior KDB goniotomy eyes, and in $64.29 \%(27 / 42)$ in goniotomy-naive eyesthis difference was not statistically significant $(p=0.58)$. Both pre-laser mean IOP and postlaser mean IOP at 6 months were comparable between treatment groups (Table 2). However, pre- to post-laser IOP reduction was only significant in the goniotomy-naive cohort $(2.50 \pm 4.0 \mathrm{mmHg} \quad[p=0.01] \quad$ vs $2.35 \pm 4.7 \mathrm{mmHg}[p=0.08])$. Medication use at 6 months and medication reduction after LTP was comparable between two groups $(p=0.78)$ (Table 3).

\section{DISCUSSION}

Since the development of ALT in the 1970s, the use of LTP for open-angle glaucoma has played a crucial role in the management of glaucoma. 
With the advent of SLT and MLT, both forms of LTP have shown promise in lowering IOP in eyes irrespective of the status of the lens $[17,18]$. A meta-analysis by Zhou et al. showed that all available types of LTP are equally effective at decreasing IOP compared to medicationbased therapies [17]. Recently, King et al. showed that the outcome of LTP is not predictive of the outcome of subsequent KDB goniotomy [10]. In this study, we sought to determine whether LTP could successfully lower IOP in eyes with prior KDB goniotomy. Given the limitation of IOP-lowering capacity of trabecular-bypassing MIGS procedures, LTP would be expected to produce suboptimal results in eyes with distal outflow obstruction despite enhanced trans-trabecular flow.

The only other study comparing success of LTP after angle surgery retrospectively examined 14 eyes that received SLT after phaco-trabectome, and they reported $42.9 \%$ success rates at 3 months and $7.7 \%$ between 8 and 10 months following SLT. However, the study did not include a control group [19]. Our results show that eyes that had prior KDB goniotomy failed to achieve significant IOP and medication reduction at 6 months after LTP.

There are multiple potential factors contributing to the comparable success rates and significant IOP reductions in the goniotomynaive group only. One potential confounder is the fact that some eyes $(38.1 \%)$ received laser trabeculoplasty prior to the KDB goniotomy. This was similar to Töteberg-Harms and Rhee [19], who also reported that $42.9 \%$ of patients received laser trabeculoplasty prior to their phaco-trabectome. However, the results of our sub-analysis show that previous LTP may not affect the outcome of a second LTP after KDB goniotomy (Supplemental Tables 1-3). Success rates, IOP reduction, and medication reduction were comparable between eyes with a history of LTP versus LTP-naive eyes that underwent prior KDB goniotomy.

Another potential confounder is that most of the patients $(85.7 \%$ in the prior KDB goniotomy group vs $73.8 \%$ in the control group) underwent MLT as their LTP procedure after KDB goniotomy. However, since both groups were matched at baseline for all demographic factors-including type of LTP-the imbalance of MLT should not be concerning for this particular study. In addition, prior studies have reported comparable IOP-lowering outcomes between MLT and SLT $[12,13]$.

The main limitation of this study is its retrospective design, and selection bias related to case selection may further affect results. For example, LTP is frequently offered in those patients whose IOP is only minimally above target (if not they would have been offered surgical intervention or a ciliary ablation laser), which may have contributed to their minimal IOP- or medication-lowering effect. In addition, since most of our patient population was Caucasian with a diagnosis of primary open-angle glaucoma, our results may be less generalizable to broader populations.

The possibility of successfully identifying potential candidates for successful laser procedures after angle surgery is intriguing. We hope that this study provides additional insight into the potential outcomes for patients with LTP following KDB goniotomy and further interest into selection criteria for patients undergoing the aforementioned procedures.

\section{CONCLUSION}

Eyes with prior KDB goniotomy had limited IOP- or medication-lowering response to subsequent LTP performed.

\section{ACKNOWLEDGEMENTS}

Funding. New World Medical provided funding for the manuscript including the Journal's Rapid Service Fees, but had no editorial control over its content.

Prior Presentation. An abstract from this study was presented at World Glaucoma Congress (WGC) 2021 and American Society of Cataract and Refractive Surgery (ASCRS) Meeting 2021. 
Authorship. All named authors meet the International Committee of Medical Journal Editors (ICMJE) criteria for authorship for this article, take responsibility for the integrity of the work as a whole, and have given their approval for this version to be published.

Authorship Contributions. All authors contributed to the study conception and design. Material preparation, data collection and analysis were performed by Junsang Cho, Daniel Hogan, Muhammad Salim, Eli Pratte. The first draft of the manuscript was written by Junsang Cho and all authors commented on previous versions of the manuscript. All authors read and approved the final manuscript.

Disclosures. Jella An MD is a consultant for New World Medical. Remaining authors have no disclosures.

Compliance with Ethics Guidelines. The study was approved by the University of Missouri-Columbia School of Medicine Institutional Review Board with a waiver of informed consent due to the retrospective nature of the study. The protocol adhered to the tenets of the Declaration of Helsinki and was compliant with HIPAA.

Data Availability. The data sets generated during and/or analyzed during the current study are available from the corresponding author on reasonable request.

Open Access. This article is licensed under a Creative Commons Attribution-NonCommercial 4.0 International License, which permits any non-commercial use, sharing, adaptation, distribution and reproduction in any medium or format, as long as you give appropriate credit to the original author(s) and the source, provide a link to the Creative Commons licence, and indicate if changes were made. The images or other third party material in this article are included in the article's Creative Commons licence, unless indicated otherwise in a credit line to the material. If material is not included in the article's Creative Commons licence and your intended use is not permitted by statutory regulation or exceeds the permitted use, you will need to obtain permission directly from the copyright holder. To view a copy of this licence, visit http://creativecommons.org/licenses/by$\mathrm{nc} / 4.0 /$.

\section{REFERENCES}

1. Heijl A, Leske MC, Bengtsson B, et al. Reduction of intraocular pressure and glaucoma progression: results from the Early Manifest Glaucoma Trial. Arch Ophthalmol. 2002;120:1268-79. https://doi. org/10.1001/archopht.120.10.1268.

2. Hirabayashi MT, King JT, Lee D, An JA. Outcome of phacoemulsification combined with excisional goniotomy using the Kahook Dual Blade in severe glaucoma patients at 6 months. Clin Ophthalmol. 2019;13:715-21.

3. Hirabayashi MT, Lee D, King JT, Thomsen S, An JA. Comparison of surgical outcomes of $360^{\circ}$ circumferential trabeculotomy versus sectoral excisional goniotomy with the Kahook Dual Blade at 6 months. Clin Ophthalmol. 2019;13:2017-24.

4. Lee D, King J, Thomsen S, Hirabayashi M, An J. Comparison of surgical outcomes between excisional goniotomy using the Kahook Dual Blade and iStent trabecular micro-bypass stent in combination with phacoemulsification. Clin Ophthalmol. 2019;13:2097-102.

5. Newman-Casey PA, Robin AL, Blachley T, et al. The most common barriers to glaucoma medication adherence: a cross-sectional survey. Ophthalmology. 2015;122(7):1308-16.

6. Schwartz GF, Hollander DA, Williams JM. Evaluation of eye drop administration technique in patients with glaucoma or ocular hypertension. Curr Med Res Opin. 2013;29(11):1515-22.

7. Diakonis V, Yesilirmak N, Cabot F, et al. Comparison of surgically induced astigmatism between femtosecond laser and manual clear corneal incisions for cataract surgery. J Cataract Refract Surg. 2015;41(10):2075-80. https://doi.org/10.1016/j. jcrs.2015.11.004.

8. Wang J, Zhang E, Fan W, Ma J, Zhao P. The effect of micro-incision and small-incision coaxial phacoemulsification on corneal astigmatism. Clin Exp Ophthalmol. 2009;37(7):664-9. https://doi.org/10. 1111/j.1442-9071.2009.02117.x. 
9. Lavia C, Dallorto L, Maule M, Ceccarelli M, Fea AM. Minimally-invasive glaucoma surgeries (MIGS) for open angle glaucoma: a systematic review and meta-analysis. PLoS ONE. 2017;12(8): e0183142. https://doi.org/10.1371/journal.pone.0183142. PMID:28850575;PMCID:PMC5574616.

10. King J, Hirabayashi M, An J. Relationship between intraocular pressure responses to selective laser trabeculoplasty and excisional goniotomy with Kahook Dual Blade combined with phacoemulsification in glaucomatous eyes. Can J Ophthalmol. 2021. https://doi.org/10.1016/j.jcjo.2021.02.040.

11. American Academy of Ophthalmology. ICD-10-CM Quick Reference Guide for Glaucoma. San Francisco: American Academy of Ophthalmology; 2015.

12. Hirabayashi MT, Rosenlof TL, An JA. Comparison of successful outcome predictors for MicroPulse ${ }^{\circledR}$ laser trabeculoplasty and selective laser trabeculoplasty at 6 months. Clin Ophthalmol. 2019;13:1001-9. https://doi.org/10.2147/OPTH.S205977.

13. De León M, Ortega Santana JF, García LA. Selective laser trabeculoplasty vs micropulse laser trabeculoplasty for the treatment of open angle glaucoma and ocular hypertension. Int J Res Med Health Sci. 2017;14(1):30-4.

14. De Keyser M, De Belder M, De Groot V. Selective laser trabeculoplasty in pseudophakic and phakic eyes: a prospective study. Int J Ophthalmol. 2017;10(4):593-598. https://doi.org/10.18240/ijo. 2017.04.15.

15. New World Medical, Inc. Kahook Dual Blade. Instructions for use. Rancho Cucamonga, CA, 2018.

16. Klamann MKJ, Gonnermann J, Maier A-KB, et al. Influence of selective laser trabeculoplasty (SLT) on combined clear cornea phacoemulsification and trabectome outcomes. Graefes Arch Clin Exp Ophthalmol. 2014;252(4):627-31.

17. Valera Cornejo D, Loayza Gamboa W, Herrera Quiroz J, et al. Micropulse laser trabeculoplasty as an adjuvant treatment for uncontrolled open angle glaucoma. New Front Ophthalmol. 2017;4(1). https://doi.org/10.15761/NFO.1000187.

18. Zhou R, Sun Y, Chen H, Sha S, He M, Wang W. Laser trabeculoplasty for open-angle glaucoma: a systematic review and network meta-analysis. Am J Ophthalmol. 2020;S0002-9394(20):30412-8. https://doi.org/10.1016/j.ajo.2020.07.046.

19. Töteberg-Harms M, Rhee DJ. Selective laser trabeculoplasty following failed combined phacoemulsification cataract extraction and ab interno trabeculectomy. Am J Ophthalmol. 2013;156(5): 936-940.e2. https://doi.org/10.1016/j.ajo.2013.05. 044. 